\title{
An Algorithm for the Second Immanant
}

\author{
By Robert Grone and Russell Merris*
}

\begin{abstract}
Let $\chi$ be an irreducible character of the symmetric group $S_{n}$. For $A=\left(a_{i j}\right)$ an $n$-by- $n$ matrix, define the immanant of $A$ corresponding to $\chi$ by

$$
d(A)=\sum_{\sigma \in S_{n}} \chi(\sigma) \prod_{t=1}^{n} a_{t \sigma(t)} .
$$

The article contains an algorithm for computing $d(A)$ when $\chi$ corresponds to the partition $\left(2,1^{n-2}\right)$.
\end{abstract}

Introduction. Denote by $\chi_{k}$ the (irreducible, characteristic zero) character of the symmetric group $S_{n}$ corresponding to the partition $\left(k, 1^{n-k}\right)$, for $k=1,2, \ldots, n$. If $A=\left(a_{i j}\right)$ is an $n$-by- $n$ matrix, define

$$
d_{k}(A)=\sum_{\sigma \in S_{n}} \chi_{k}(\sigma) \prod_{t=1}^{n} a_{t \sigma(t)} .
$$

Then, for example, $d_{1}(A)=\operatorname{det}(A)$ and $d_{n}(A)=\operatorname{per}(A)$, the permanent of $A$. In general, $d_{k}$ is known as an immanant or a generalized matrix function. (An immanant is a generalized matrix function based on $S_{n}$.)

Suppose $G$ is a (simple) graph on $n$ vertices. Denote by $L(G)$ the Laplacian matrix corresponding to some labeling of the vertices of $G$, i.e., $L(G)$ is an $n$-by- $n$ matrix, the $(i, j)$ entry of which is the degree of vertex $i$ when $i=j,-1$ if $i \neq j$ but vertex $i$ is adjacent to vertex $j$, and zero otherwise. It is shown in [5] that the number of Hamiltonian circuits in $G$ is given by the formula

$$
h(G)=\frac{1}{2 n} \sum_{k=2}^{n}(-1)^{k} d_{k}(L(G)) .
$$

While there is an immense literature on generalized matrix functions, Eq. (1) is already sufficient motivation to seek "fast" algorithms for their actual computation. The main result of this note is an algorithm for computing $d_{2}$. (See the next section.)

It seems that $d_{2}$ may be especially appropriate for the study of Laplacian matrices for the following reason: If $G$ is a graph on $n$ vertices, then $L(G)$ is positive semidefinite symmetric and singular. Moreover, $G$ is connected if and only if rank $L(G)=n-1$. For arbitrary positive semidefinite symmetric matrices without a zero row, it was established in [3, Corollaries 5 and 6] that $d_{2}(A) \geqslant 0$ with equality if and only if $\operatorname{rank}(A)<n-1$.

Received August 22, 1983.

1980 Mathematics Subject Classification. Primary 65F30, 15A15, 05 C50.

*Work of the second author was supported by the National Science Foundation under Grant No. MCS-8300097. 
Two graphs, $G_{1}$ and $G_{2}$ are isomorphic if and only if $L\left(G_{1}\right)$ is permutation similar to $L\left(G_{2}\right)$. Thus, any permutation-similarity invariant of $L(G)$ is actually a property of the underlying graph $G$. This observation has motivated the use of determinants in the study of graphs. (See, e.g. [1].) The trouble with determinants is that they are preserved under arbitrary similarities. It was suggested in [6] that permanents might be substituted for determinants. The trouble with permanents is their computational intractability. (See [7]. It is already evident from (1) that the computation of at least some immanants is as hard as the Hamiltonian circuit problem.) It occurred to us that $d_{2}$ might afford a reasonable compromise, and this turns out to be the case.

The key to fast algorithms for $d_{1}$ lies in the observation that the determinant of a matrix with two equal rows is zero. If $A$ has $k+1$ equal rows. it turns out [3, Corollary 4] that $d_{k}(A)=0$. Although our algorithm does not make explicit use of this observation, it provides some evidence (corroborated by Werner Hartmann [2]) that $d_{k}(A)$ should become easier to compute as $k$ decreases. On the other hand, there may be something unique about $d_{2}$. It was shown in [4] that $d_{2}$, but not $d_{k}$ for $k>2$, is linked in an interesting way with the determinant, namely $d_{2}(A) \operatorname{det}\left(A^{-1}\right)$ $=d_{2}\left(A^{-1}\right) \operatorname{det}(A)$, for all invertible matrices $A$.

An Algorithm for $d_{2}$. If $\sigma \in S_{n}$, then $\chi_{2}(\sigma)=\varepsilon(\sigma)(F(\sigma)-1)$, where $\varepsilon$ is the alternating (signum) character and $F(\sigma)$ is the number of fixed points of $\sigma$. It follows from this fact that $d_{2}(A)=-\operatorname{det}(A)$ for any matrix $A$ with main diagonal consisting entirely of zeros. Our algorithm is based on this observation. We proceed with its description.

1. Scale $A$. Denote by $D$ the $n$-by- $n$ diagonal matrix; the $(i, i)$ entry of which is 1 if $a_{i i}=0$ and $1 / a_{i i}$ if $a_{i i} \neq 0$. Let $A_{1}=D^{-1} A$. Then $d_{2}(A)=\operatorname{det}(D) d_{2}\left(A_{1}\right)$.

2. Permutation Similarity. (While this step is unnecessary, it makes the subsequent discussion easier to follow.) If $A$ has $r$ main diagonal elements equal to zero, let $P$ be a permutation matrix such that the first $r$ main diagonal elements of $A_{2}=P^{t} A_{1} P$ are zero. Then $d_{2}\left(A_{1}\right)=d_{2}\left(A_{2}\right)$.

3. Polynomial Coefficients. At this point, we are dealing with a matrix, $A_{2}$, whose main diagonal begins with $r$ (possibly $r=0$ ) zero entries. The remaining $n-r$ entries are ones. Imagine replacing each 1 with an indeterminate $\lambda$. Call the resulting matrix $\Lambda$. Then $\Lambda=\operatorname{diag}(0, \ldots, 0, \lambda, \ldots, \lambda)+E$, where the $(i, j)$ entry of $E$ is equal to the $(i, j)$ entry of $A_{2}$ provided $i \neq j$; and each diagonal entry of $E$ is zero. Denote the polynomial $\operatorname{det}(\Lambda)$ by $c_{r} \lambda^{n-r}+\cdots+c_{n-1} \lambda+c_{n}$. Consider the coefficient $c_{k}$. For $k=0, c_{0}$ is 0 or 1 ; it is 1 if and only if $r=0$, i.e., if and only if $\Lambda=\lambda I_{n}+E$. Observe that $c_{1}$ is always 0 , for no permutation of $S_{n}$ has exactly $n-1$ fixed points. For $k>1, c_{k}$ is the sum of the determinants of all $k$-by- $k$ principal submatrices of $E$ which contain the leading $r$-by-r principal submatrix. We may formalize this statement as follows: For $r \leqslant k \leqslant n$, let $Q_{k, n}^{r}$ denote the set of strictly increasing functions $\alpha$ : $\{1,2, \ldots, k\} \rightarrow\{1,2, \ldots, n\}$ such that $\alpha(i)=i$ for $i=1,2, \ldots, r$. Then (for $k>1$ ),

$$
c_{k}=\sum_{\alpha \in Q_{k, n}^{r}} \operatorname{det}(E[\alpha])
$$

where $E[\alpha]$ is the $k$-by- $k$ principal submatrix of $E$ corresponding to rows and columns $\alpha$, i.e., the $(i, j)$ entry of $E[\alpha]$ is the $(\alpha(i), \alpha(j))$ entry of $E$. 
Now, the indeterminate $\lambda$ is merely a device to help us organize together those diagonal products of $A_{2}$ which involve exactly $n-k$ fixed points, none of which corresponds to a main diagonal zero. That is,

$$
c_{k}=\sum \varepsilon(\sigma) \prod_{t=1}^{n} a_{t \sigma(t)}^{(2)},
$$

where $A_{2}=\left(a_{i j}^{(2)}\right)$, and where the summation is over all permutations $\sigma \in S_{n}$ which fix exactly $n-k$ of the integers $\{r+1, r+2, \ldots, n\}$ and none of the integers $\{1,2, \ldots, r\}$. To achieve $d_{2}\left(A_{2}\right)$, it remains to multiply each diagonal product in (3) by $F(\sigma)-1=n-k-1$, and sum on $k$. Thus,

$$
d_{2}(A)=\operatorname{det}(D) \sum_{k=0}^{n}(n-k-1) c_{k},
$$

where $c_{k}$ is given by (2).

Of course, the more zeros $A$ has on its main diagonal, the faster the algorithm works. At worst, it is comparable to the evaluation of the characteristic polynomial.

Department of Mathematics

Auburn University

Auburn, Alabama 36849

Department of Mathematics and Computer Science

California State University

Hayward, California 94542

1. D. M. Cvetkovic, M. Doob \& H. Sachs, Spectra of Graphs, Academic Press, New York, 1980.

2. W. HARTMANN, private communication.

3. R. MERRIS, “On vanishing decomposable symmetrized tensors," Linear and Multilinear Algebra, v. 5 , 1977, pp. 79-86.

4. R. MERRIS, "Representations of $G L(n, R)$ and generalized matrix functions of class MPW," Linear and Multilinear Algebra, v. 11, 1982, pp. 133-141.

5. R. MERRIS, "Single-hook characters and Hamiltonian circuits," Linear and Multilinear Algebra, v. 14, 1983, pp. 21-35.

6. R. MERRIS, K. R. ReBman \& W. Watkins, "Permanental polynomials of graphs," Linear Algebra Appl., v. 38, 1981, pp. 273-288.

7. L. G. Valiant, “The complexity of computing the permanent," Theoret. Comput. Sci., v. 8, 1979, pp. 189-201. 